Bangladesh J. Bot. 42(2): 321-325, 2013 (December)

\title{
CAFFEINE, POLYPHENOL AND CRUDE FAT CONTENTS IN TEA VARIETIES AVAILABLE IN BANGLADESH
}

\author{
GM Rabiul Islam*, Muhammad Intizer Nabi, Md Mozammel Hoque \\ AND Abu Yusuf ${ }^{1}$ \\ Department of Food Engineering and Tea Technology, Shahjalal University of Science \\ and Technology, Sylhet-3114, Bangladesh
}

Key words: Bangladesh, Tea varieties, Caffeine, Polyphenol, Crude fat content

\begin{abstract}
The total caffeine content in Bangladesh Tea (BT) varieties (BT1-BT18) ranged from $1.59 \pm 0.155$ $4.15 \pm 0.399 \mathrm{~g} / 100 \mathrm{~g}$ of $\mathrm{DM}$ and the crude fat content in BT varieties varied from $6.69 \pm 0.64-9.87 \pm 0.9509$ $\mathrm{g} / 100 \mathrm{~g}$ of dry material (DM). In this study, the highest amount of caffeine (4.15 $\pm 0.399 \mathrm{~g} / 100 \mathrm{~g} \mathrm{DM})$ was found in BT-16 variety $(\mathrm{p}<0.05)$ while the highest amount of crude fat $(9.24 \pm 0.889 \mathrm{~g} / 100 \mathrm{~g} \mathrm{DM})$ was found in BT 10 variety $(\mathrm{p}>0.5)$. The total polyphenol content ranged:8.66 $\pm 0.831-14.89 \pm 1.432 \mathrm{GAE}$; g/100 g DM. The highest amount of polyphenol was found in BT 9 variety $(14.890 \pm 1.432 \mathrm{GAE} ; \mathrm{g} / 100 \mathrm{~g}$ DM) $(\mathrm{p}<$ 0.05).
\end{abstract}

\section{Introduction}

Caffeine and polyphenols are the predominant factor for assuring the quality of tea (Camellia sinensis L. Kuntze) leaf (Chen 1999). Caffeine (1,3,7-trimethylxanthene) is bitter, white crystalline purine derived alkaloid that acts as a stimulant drug (Eggers et al. 2001) and is a diverse group of compounds that are found primarily in plants and contain basic nitrogen (Barone et al. 1996; Frary et al. 2005). Among the alkaloids probably the most physiologically active constituent is caffeine being principal representative at a level of 1 - 5\% of its dry weight (Stagg and Millin 1975). It is the most powerful xanthine, in its ability to increase alertness, put off sleep and to improve attention. It also acts as a vasodilator (relaxes the blood vessels) as well as a diuretic (increase urination) (Bolton 1981).

The polyphenols (Mukhtar et al. 2000) in tea mainly include flavanols, hydroxyl-4-flavanols, anthocyanins, flavones and phenolic acids which have a synergestic effect involving caffeine to (Stagg and Millin 1975) contribute the bitterness, astringency and sweetness of tea (Hara et al. 1995). Polyphenols are widely used for prophylaxis and treatment of a variety of disorders. Lipid is also necessary though lipids are not major constituents in a tea brew but they can play an important roles in the development of aroma (Bhuyan et al. 1989, Hara et al. 1995, Scharbert and Hofmann 2005, Wang and Ruan 2009) and has an impact on nutritional profile of tea.

however, there is no absolute measurement of caffeine, polyphenol and crude fat content of the released BT varieties. By analyzing caffeine, polyphenols and crude fat of these BT varieties, we can understand which variety is better in comparison with other varieties in order to improve agronomical characteristics through gene isolation, biotechnology, breeding and other allied fields. It will help the tea consumers to find the best quality tea from a very early civilization in relation to their financial ability (Islam et al. 2005, Choudhury 2010) as the tea consumers have a demand. Hence, the objective of the study is to evaluate the amount of caffeine, polyphenol and crude fat content of BT varieties to understand which BT variety is better in comparison with other varieties for cultivation and commercialization.

*Author for correspondence: <rabi-ttc@sust.edu>. ${ }^{1}$ Department of Chemical Engineering and Polymer Science, Shahjalal University of Science and Technology, Sylhet-3114, Bangladesh. 


\section{Materials and Methods}

Leaf samples were collected from 18 varieties of tea (Camelia sinensis L. Kuntze, Fam.: Theaceae) released by the Bangladesh Tea Research Institute (BTRI), Srimangal, Moulvi Bazar. The name of the varieties ranged from BT1 - BT18, each having different agronomical characteristics. For sampling, plants from the Germplasm Centre of BTRI were used. After collection the samples were sun dried to keep the biochemical properties in good shape (Choudhury 2010). The sun dried material was crushed and/or ground to powder form for further analysis. Solvent extraction method was used for the extraction of tea (AACC 2000). Water soluble compounds were extracted from dry leaves with boiling water followed by another extraction from water into organic solvent. At last, the solvent was removed and the crude material was extracted.

The caffeine and crude fat content was estimated after (AACC 2000) and the total polyphenol content (TPC) was determined according to the method described by the International Organization for Standardization (ISO 14502-1:2005) using gallic acid as standard. At each determination of a parameter, three replicate samples were used. Data were expressed as the means \pm standard error of the mean (SEM) of three independent experiments. Variance analysis, with a significance level of 'alpha' $=0.05 \%$, was performed to determine the differences in the experimental content while the Turkey methods were applied for multiple comparisons. The analyses were performed using the SPSS package version 17.

\section{Results and Discussion}

The content of caffeine, polyphenol and crude fat of different Bangladesh Tea (BT) varieties varied significantly $(p=0.00)$. Caffeine content rangedfrom $1.59 \pm 0.155$ to $4.15 \pm 0.399 \mathrm{~g} / 100 \mathrm{~g}$ DM (Fig. 1). These results were similar to the findings of Amra et al. (2006) and Bennett and Bonnie (2001). In the present study, the maximum content of caffeine was found in BT16 variety (4.15 $\pm 0.399 \mathrm{~g} / 100 \mathrm{~g} \mathrm{DM})$. Other varieties such as BT14, BT17 and BT18 showed $\mathrm{n}$ approximately similar amount of caffeine content $(\mathrm{p}<0.05)$. BT1 varieties contains lower amount of caffeine and no significant variation was observed between BT1 and BT3 varieties because of their homogenous nature in comparison with other varieties $(p>0.05)$. The variation of the other reported values may be due to varietal defference , growing environment, agronomical practices, etc. in the field as each influences the tea leaf and final infusion compositions (Conrad et al. 2001).

On the other hand Polyphenol content ranged from $8.66 \pm 0.831$ to $14.89 \pm 1.432$ (GAE; g/100 g DM) (Fig. 2). Lin et al. (2003) reported that tea leaves contain high phenolic components which account for 25 - 35\% on dry weight basis. Again they argued that soluble polyphenols constitute about $15 \%$ of black tea but this variation may occur due to the different variety of tea, its geographical origin, environmental conditions and agronomic situations (Conrad et al. 2001). As the contents of polyphenol found in this study were low in comparison to Lin et al. (2003) this may be due to the fact of oxidation of flavanols, flavandiols and theogallin during the conversion of fresh leaf to the black tea (Stagg and Millin 1975, Robertson 1992). In this study, we found that, BT9 contributes higher amount of polyphenols (14.890 $\pm 0.950 \mathrm{GAE}$; g/100 $\mathrm{DM})$. Besides, no significant variation was found between the polyphenol content of BT9 and BT16 varieties because of their homogenous nature in comparison with other varieties ( $p>0.05)$. In this analysis, BT17 shows significant variation in polyphenol content $(8.660 \pm 0.831 \mathrm{~g} / 100 \mathrm{~g}$ DM) which is the lowest of all. 


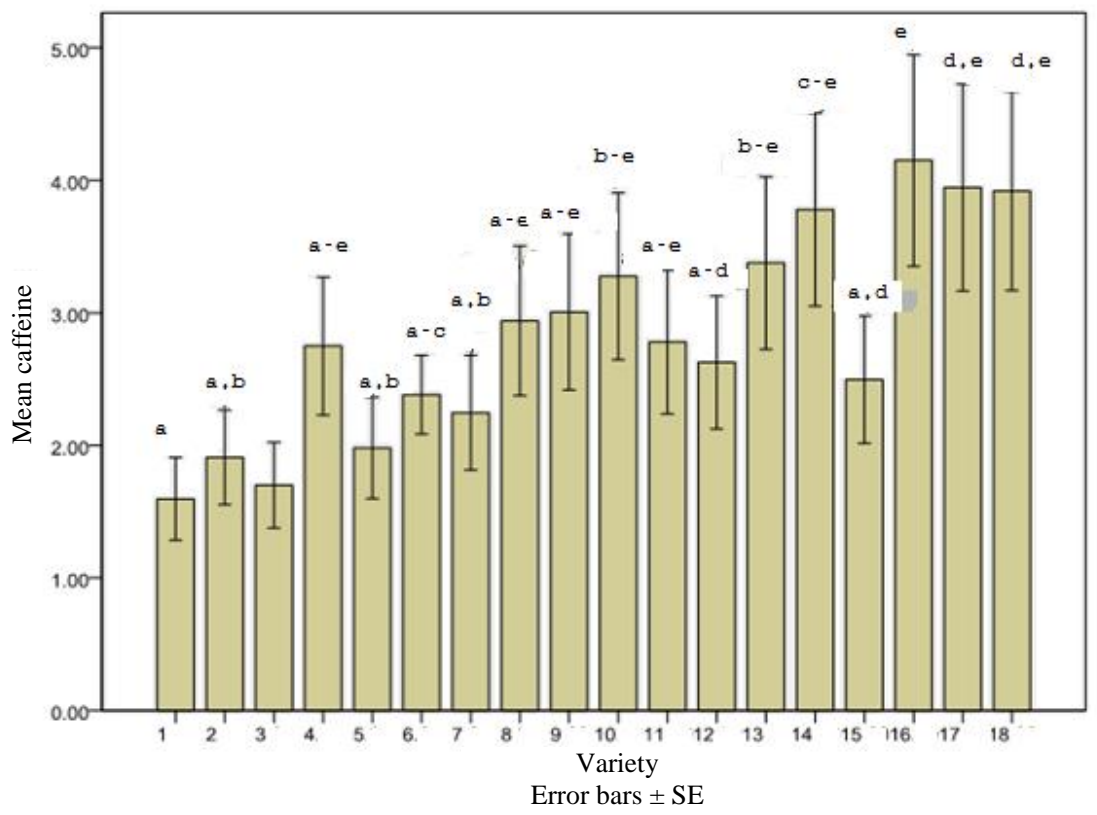

Fig. 1. Total caffeine content (g/100 g DM) in different BT varieties (BT1-BT18). Bars represent the Mean \pm SEM), different letters (a-e) denote that the significant difference were observed in caffeine content in different varieties) $(\mathrm{p}<0.05)$.

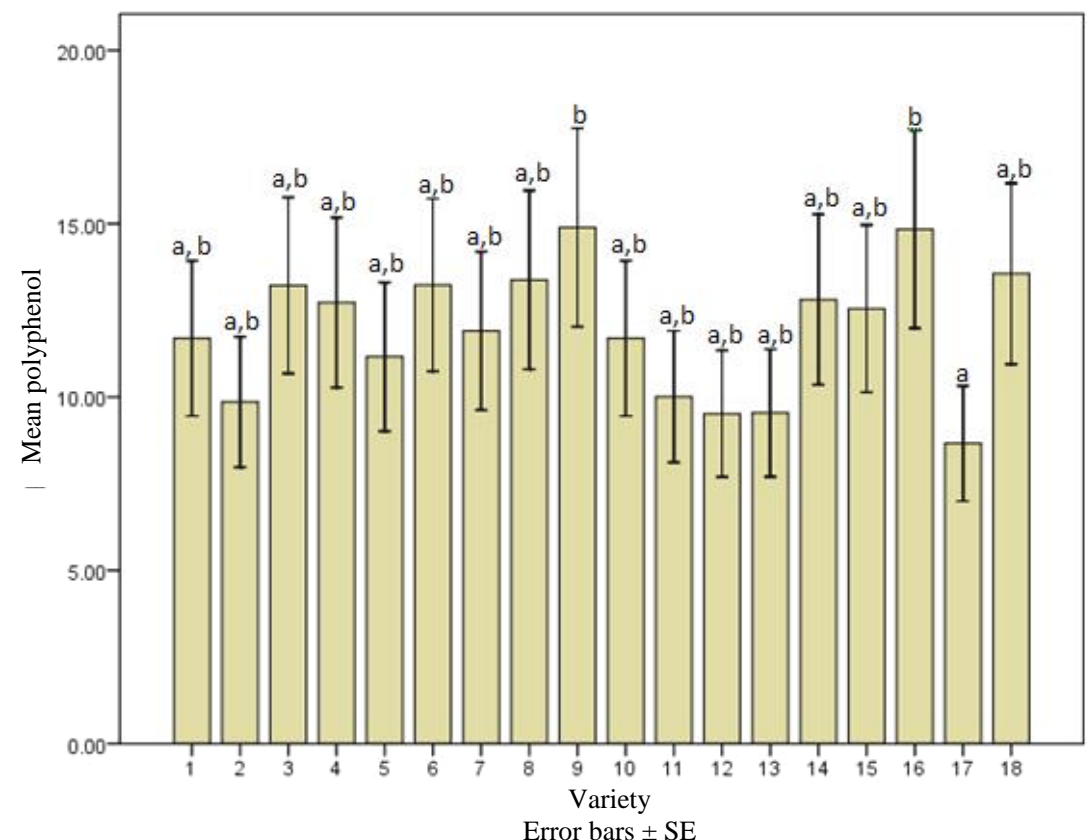

Fig. 2. Total polyphenol content expressed as (g/100 g DMial) in different varieties (BT1 - BT18). (Bars represent the Mean \pm SEM, different letters $(a, b)$ denote the level of significance $(p<0.05)$. 
No significant differences were observed among the varieties $(\mathrm{p}=0.07)$ in respect of crude for content. The fat content ranged from $6.69 \pm 0.64$ to $9.87 \pm 0.9509$ g/100g DM (Fig. 2). Extended research was conducted in the similar field (Hara et al. 1995, Millin and Rustidge 1967). According to Hara et al. (1995), the fat content of green leaf varies from 7 - 10\% of its dry weight. While according to Millin and Rustidge (2000) the fat present in the protoplasm up to 3-7\% of its dry weight that coincides with the present study. The maximum content of fat was found in BT17 variety $(9.24 \pm 0.889 \mathrm{~g} / 100 \mathrm{~g} \mathrm{DM})$ and the lowest in BT4 (6.28 $\pm 0.600 \mathrm{~g} / 100 \mathrm{~g} \mathrm{DM})$.

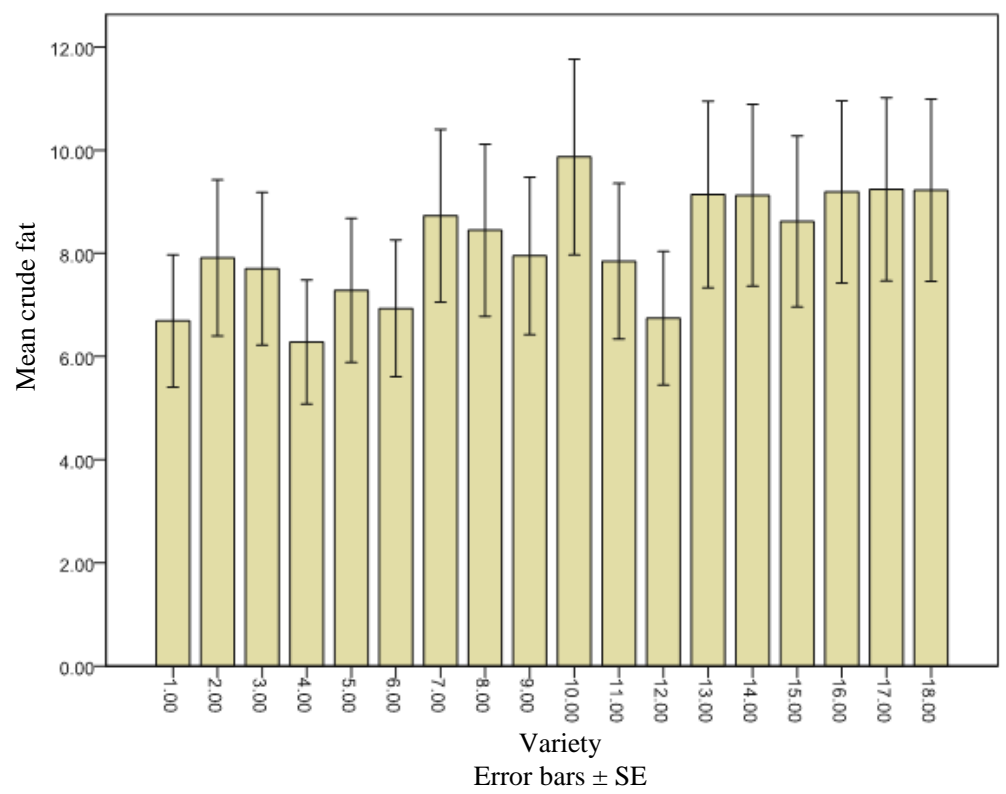

Fig. 3. Total crude fat content (g/100 g DM) in different BT varieties. Numbers (1-18) denotes Bangladesh tea varieties. Bars represent the Mean \pm SEM, no significant variation was observed in different varieties of crude fat $(\mathrm{p}<0.5)$.

Through this study, it was apparent the BT16 and BT9 contribute higher amount of caffeine and polyphenol content. In contrast, BT1 and BT17 contain lower amount of caffeine and polyphenol content. Crude fat was also analyzed and found that BT10 contains higher amount of crude fat. As consumption of tea is gaining popularity all over the world, scientists of Bangladesh need to produce quality clone tea rich in bioactive compounds for successful commercialization.

\section{Acknowledgement}

The support extended by Bangladesh Tea Research Institute (BTRI) to carry out the present study is duly acknowledged.

\section{References}

AACC 2000. Approved Methods of American Association of Cereal Chemists. Am. Assoc. Cereal Chem Inc. St. Paul. Minnesota.

Barone JJ and Roberts HR 1996. Caffeine consumption. Food Cosmet Toxicol. 34: 119-129. 
Bhuyan LP, Tamuly P and Mahanta PK 1989. Studies on fatty acids composition in tea (Camellia sinensis). J. Sci. Food Agric. 46: 325-330.

Bolton S and Null G 1981. Caffeine: Psychological Effects, Use and Abuse. Orthomolecular Psychiatry. 10(3): $202-211$.

Chen Z 1999. Pharmacological functions of tea. In: Global Advances in Tea Science. Ed. Jain, N.K. 333-358, Aravali Books International (Pvt.) Ltd., India.

Choudhury A 2010. Optimum quality Teas processing and pharmaceutical uses. S Ahmad, Srimangal, Bangladesh. 2, 21-27, 29, 30,155-160.

Conrad A, Birch MR, Dacombe C, Humphrey PG and Martin PT 2001. Factors affecting the caffeine and polyphenol contents of black and green tea infusions. J. Agric. Food Chem. 49: 5340-5347.

Eden T 1958. The development of tea culture. In: Tea. Ed. Eden, T, Longman, London. 1-4.

Eggers H, Cnattingius S, Signorello LB and Anneren G 2001. Caffeine intake and the risk of first-trimester spontaneous abortion. N. Engl. J. Med. 343: 1839-1845.

Frary CD, Johnson RK, and Wang MQ 2005. Food sources and intakes of caffeine in the diets of persons in the United States. J Am Diet Assoc. 105: 110-113.

Graham H N 1999. In Wiley Encyclopedia of Food Science and Technology, 2nd ed.; Frederick, J. F., Ed.; John Wiley and Sons: New York. 1-4: 2292-2305.

Hara Y, Luo S J, Wickremasinghe R L and Yamanishi T 1995. Special issue on tea. Food Rev. Int. 11: 371545.

Islam GMR, Iqbal M, Quddus K, and Ali. MY, 2005. Present status and future needs of tea industry in Bangladesh. Proc. Pakistan Acad. Sci. 42(4): 305-314.

ISO 14502-1: 2005. Determination of substances characteristic of green and black tea. Part 1: Content of total polyphenols in tea. Colorimetric method using Folin-Ciocalteu reagent.

Lin YI, Juan I M , Chen Yl, Liang YC and Lin J K 2003. Composition of polyphenols in fresh tea leaves J. Agric. Food Chem. 44: 1387-1394.

Millin DJ and Rustidge DW 1967. Tea manufacture. Process Biochem. 6: 9-13.

Mukhtar H and Ahmad N 2000. Tea polyphenols: prevention of cancer and optimizing health. Am. J. Clin. Nut. 71: 1698-1702.

Robertson A 1992. The chemistry and biochemistry of tea productions the nonvolatiles. In: Teas cultivation to consumption; Willson, K. C., Clifford, M. N., Eds.; Chapman and Hall: London, U.K. 555-601.

Scharbert S and Hofmann T 2005. Molecular definition of black tea taste by means of quantitative. studies tastes reconstitution and omission experiments. J. Agric. Food Chem.53: 5377-5384.

Stagg GV and Millin DJ 1975. The nutritional and therapeutic value of tea-a review, J. Sci. Food Agr. 26: 590-1439.

Wang KB and Ruan JY 2009. Analysis of chemical components in green tea in relation with perceived quality. Int. J. food Sci. Tech. 44: 2476-2484.

(Manuscript received on 18 November, 2012; revised on 4 April, 2013) 